\title{
Extended Rectangular Drawings of Plane Graphs with Designated Corners (Extended Abstract)
}

\author{
Kazuyuki Miura, Ayako Miyazawa, and Takao Nishizeki \\ Graduate School of Information Sciences \\ Tohoku University, Sendai 980-8579, Japan \\ \{miura,miyazawa\}@nishizeki.ecei.tohoku.ac.jp \\ nishi@ecei.tohoku.ac.jp
}

\begin{abstract}
In a rectangular drawing of a plane graph, each edge is drawn as a horizontal or vertical line segment, and all faces including the outer face are drawn as rectangles. In this paper, we introduce an "extended rectangular drawing" in which all inner faces are drawn as rectangles but the outer face is drawn as a rectilinear polygon with designated corners, and give a necessary and sufficient condition for a plane graph to have an extended rectangular drawing.
\end{abstract}

\section{Introduction}

Automatic drawing of graphs has recently created intense interest due to their broad applications in VLSI floorplanning, computer network, software design etc. [BETT99.BS88 GT97.L90.RNN02.RNN98]. In this paper, we introduce an "extended rectangular drawing" of a plane graph, which is a generalization of a "rectangluar drawing," and obtain a characterization of plane graphs having extended rectangular drawings.

In a rectangular drawing of a plane graph $G$, each edge is drawn as a horizontal or vertical line segment, and all faces including the outer face are drawn as rectangles, as illustrated in Fig. 1(a). Rectangular drawings have applications in VLSI floorplanning. The outer boundary of a VLSI chip is not always a rectangle but is often a rectilinear polygon of $\mathrm{L}-, \mathrm{T}$-, and $\mathrm{X}$-shape, and hence it is desired to find a floorplan of a VLSI chip where each "module" is assigned a rectangular space inside a rectilinear polygon [KK84 L90]. VLSI floorplans with outer boundary of L-, T-, and X-shape are illustrated in Fig. 1. Each vertex which we wish to draw as a "convex corner" of the outer face is drawn by a white circle in Fig. 1, each vertex which we wish to draw as a "concave corner" is drawn by a white circle with $\times$, each of the other vertices on the outer face is drawn by a black circle, and all vertices not on the outer face are drawn by gray circles. We call a drawing an extended rectangular drawing (with designated corners) if all inner faces are drawn as rectangles and the outer face is drawn as such a rectilinear polygon with designated corners. The maximum degree of a graph $G$ is denoted by $\Delta$. Thomassen obtained a necessary and sufficient condition for 


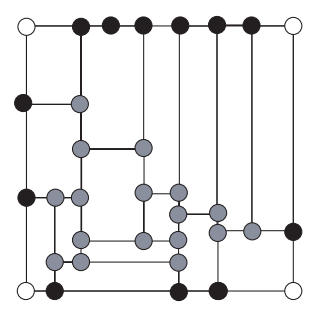

(a) Rectangular outer face

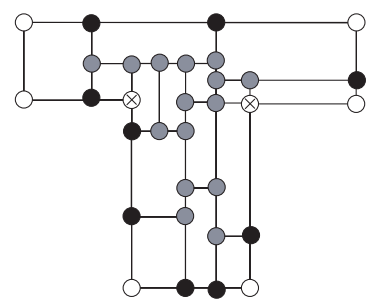

(c) T-type outer face

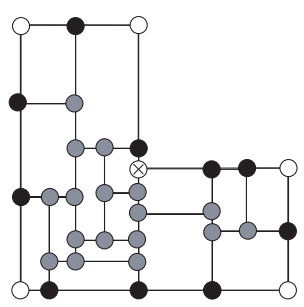

(b) L-type outer face

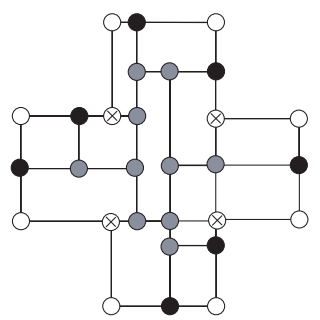

(d) X-type outer face

$\mathrm{OR}$ (convex corner)

- $S$ (non-corner)

$\otimes L$ (concave corner)

inner vertex

Fig. 1. Extended rectangular drawings.

the existence of a rectangular drawing of a plane graph $G$ with $\Delta \leq 3$ [T84], and linear-time algorithms were obtained for finding a rectangular drawing of $G$ [BS88,H93.KH97.KK84.RNN98.RNN02]. However, for a general plane graph $G$ which does not necessarily satisfy $\Delta \leq 3$, a necessary and sufficient condition has not been known for the existence of a rectangular drawing or an extended rectangular drawing. Of course, $\Delta \leq 4$ if $G$ has a rectangular drawing or an extended rectangular drawing.

As a main theorem of the paper, we give a necessary and sufficient condition for a plane graph to have an extended rectangular drawing. It immediately yields a necessary and sufficient condition for a plane graph of $\Delta \leq 4$ to have an ordinary rectangular drawing. Thomassen's condition [T84] can be immediately obtained from our main theorem as a corollary. The necessity of our condition is trivial, while the sufficiency is not. Our constructive proof of the sufficiency is outlined as follows. Let $G$ be a plane graph satisfying our condition. We choose an appropriate inner face $F$ of $G$ called a "corner face," and draw $F$ as a rectangle as illustrated in Figs. 2(a) and (b). Let $G^{*}=G-F$ be a graph obtained from $G$ by "trimming off" the face $F$, then $G^{*}$ satisfies our condition. We now choose a corner face of $G^{*}$, and draw the face as a rectangle. Repeating the operation, we eventually obtain an extended rectangular drawing of the whole graph $G$. 

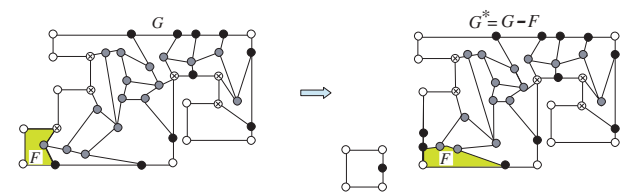

(a)
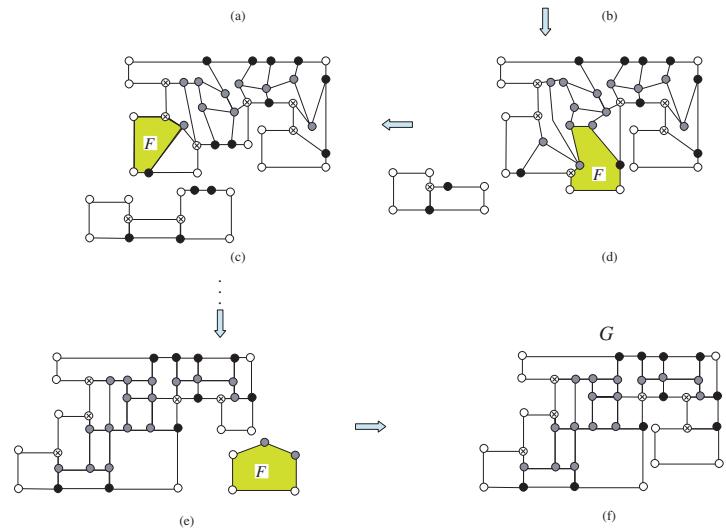

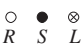

Fig. 2. The outline for a proof of the sufficiency of our main Theorem.

The remainder of the paper is organized as follows. In Section 2, we give some definitions and lemmas. In Section 3, we give a necessary and sufficient condition for a plane graph to have an extended rectangular drawing. Finally we conclude in Section 4.

\section{Preliminaries}

In this section we give some definitions and lemmas. Let $G$ be a simple connected undirected graph having no multiple edge or loop. The degree of a vertex $v$ in $G$ is the number of neighbors of $v$ in $G$, and is denoted by $d(v)$. We call a vertex of $G$ a cut vertex if its removal results in a disconnected graph. A graph $G$ is biconnected if $G$ has no cut vertex.

A plane graph $G$ is a graph embedded in the plane so that no two edges intersect geometrically except at a vertex to which they are both incident. In this paper, we assume that a plane embedding of $G$ is fixed. If a plane graph $G$ has a rectangular drawing or an extended rectangular drawing, then clearly $G$ is biconnected and $\Delta \leq 4$. We thus assume that $G$ is a plane biconnected graph with $\Delta \leq 4$. $G$ divides the plane into connected regions called faces. The contour of a face is a cycle of $G$, and is often called simply a face. We denote the outer face of $G$ by $F_{\mathrm{o}}$. A vertex and an edge of $F_{\mathrm{o}}$ is called an outer vertex and an outer edge, respectively. A vertex and an edge not on $F_{\mathrm{o}}$ is called an inner vertex and an inner edge, respectively. 
A drawing $D(G)$ of $G$ is called an extended rectangular drawing of a plane graph $G$ if

(a) each vertex of $G$ is drawn as a point in $D(G)$;

(b) each edge of $G$ is drawn as a horizontal or vertical line segment in $D(G)$;

(c) each inner face of $G$ is drawn as a rectangle in $D(G)$; and

(d) the outer face of $G$ is drawn as a rectilinear polygon in $D(G)$.

In an extended rectangular drawing $D(G)$, each cycle $C$ of $G$ is drawn as a rectilinear polygon, which is denoted by $D(C)$. A vertex of polygon $D(C)$ is a vertex of graph $G$, and is called a corner of a cycle $C$. The inner angle of a corner is either $\pi / 2$ or $3 \pi / 2$. A corner of inner angle $\pi / 2$ is called a convex corner of a cycle $C$, while a corner of inner angle $3 \pi / 2$ is called a concave corner of $C$. A vertex $v$ of $C$ is called a non-corner of $C$ if $v$ is not a vertex of polygon $D(C)$.

In this paper, we assume that a rectilinear polygonal drawing $D\left(F_{\mathrm{o}}\right)$ of the outer face $F_{\mathrm{o}}$ is roughly given: each outer vertex is designated as a convex corner, a concave corner, or a non-corner of $F_{\mathrm{o}}$. A vertex designated as a convex corner of $F_{\mathrm{o}}$ is called an $R$-vertex of $F_{\mathrm{o}}$, a vertex designated as a concave corner is called an $L$-vertex of $F_{\mathrm{o}}$, and a vertex designated as a non-corner is called an $S$-vertex of $F_{\mathrm{o}}$. (See Fig. 3(a).) If we turn around $F_{\mathrm{o}}$ clockwise, then we turn right at an $R$-vertex, turn left at an $L$-vertex, and go straight through an $S$-vertex. An extended rectangular drawing $D(G)$ is called an extended rectangular drawing with designated corners if all $R$-vertices of $F_{\mathrm{o}}$ are drawn as convex corners of $F_{\mathrm{o}}$, all $L$-vertices as concave corners, and all $S$-vertices as non-corners, as illustrated in Fig. 3(b). In this paper, we give a necessary and sufficient condition for a plane graph $G$ to have an extended rectangular drawing with designated corners. Thomassen gave a necessary and sufficient condition for a plane graph of $\Delta \leq 3$ to have a rectangular drawing [T84]. His condition corresponds to our condition for the special case where exactly four of the outer vertices are designated as $R$-vertices and all the other outer vertices are designated as $S$-vertices as illustrated in Fig. 1(a). Therefore his condition can be immediately derived from our condition.

The drawings in Figs. 1(a) and (b) are an rectangular drawing and an extended rectangular drawing of the same graph. Thus the same graph may have various extended rectangular drawings. On the other hand, the plane graph in Fig. 3(a) has an extended rectangular drawing in Fig. 3(b), but has no rectangular drawing because there is an outer vertex of degree four.

An extended rectangular drawing $D(G)$ satisfies the following four Conditions (i)-(iii) for any outer vertex $v$ of $F_{\mathrm{o}}$ :

(i) if $d(v)=2$, then $v$ is a convex corner or a non-corner of $F_{\mathrm{o}}$;

(ii) if $d(v)=3$, then $v$ is a concave corner or a non-corner of $F_{\mathrm{o}}$; and

(iii) if $d(v)=4$, then $v$ is a concave corner of $F_{\mathrm{o}}$.

We may thus assume without loss of generality that each outer vertex $v$ of $G$ is designated as follows: 


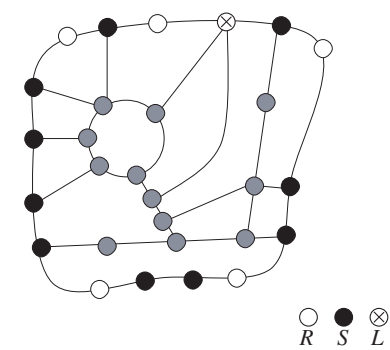

(a) A plane graph with designated outer corners

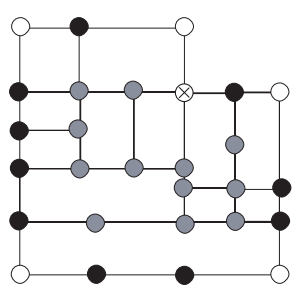

(b) An extended rectangular drawing

Fig. 3. An extended rectangular drawing.

(O-1) if $d(v)=2$, then $v$ is either an $R$-vertex or an $S$-vertex of $F_{\mathrm{o}}$;

$(\mathrm{O}-2)$ if $d(v)=3$, then $v$ is either an $L$-vertex or an $S$-vertex of $F_{\mathrm{o}}$; and

$(\mathrm{O}-3)$ if $d(v)=4$, then $v$ is an $L$-vertex of $F_{\mathrm{o}}$.

According to the designation of outer vertices, a labeling $\lambda$ of graph $G$ is defined as follows.

[Definition 1] A labeling $\lambda$ of $G$ assigns $R, S, L, R^{\prime}$ or $L^{\prime}$ as a label $\lambda(C, v)$ to each pair of a cycle $C$ of $G$ and a vertex $v$ of $C$, as follows.

Case 1: both of the two edges $e_{1}$ and $e_{2}$ of $C$ incident to $v$ are outer edges. (See Fig. 4.)

Let $\lambda(C, v)$ be the same as the designation of $v$ for $F_{\mathrm{o}}$ :

$$
\lambda(C, v)=\left\{\begin{array}{l}
R \text { if } v \text { is designated as an } R \text {-vertex of } F_{\mathrm{o}} \\
S \text { if } v \text { is designated as an } S \text {-vertex of } F_{\mathrm{o}} \\
L \text { if } v \text { is designated as an } L \text {-vertex of } F_{\mathrm{o}}
\end{array}\right.
$$

In Fig. 4 a label $\lambda(C, v)$ is written in cycle $C$; $v$ is designated as an $R$-vertex of $F_{\mathrm{o}}$ in Fig. 4(a), as an $S$-vertex in Fig. 4(b), and as an $L$-vertex in Fig. 4(c).

Case 2: both $e_{1}$ and $e_{2}$ are inner edges, and $d(v)=2$. (See Fig. 5.)

In this case, $v$ is an inner vertex. Let $\lambda(C, v)=S$. (That is, $e_{1}$ and $e_{2}$ must be drawn on the same straight line.)

Case 3: both $e_{1}$ and $e_{2}$ are inner edges, and $d(v)=3$. (See Fig. 6.)

In this case, $v$ is an inner vertex. Let $e_{3}$ be the edge incident to $v$ other than $e_{1}$ and $e_{2}$, then $\lambda(C, v)$ is defined as follows:

$$
\lambda(C, v)=\left\{\begin{array}{l}
R^{\prime} \text { if } e_{3} \text { is outside } C \\
L^{\prime} \text { if } e_{3} \text { is inside } C
\end{array}\right.
$$

Label $R^{\prime}$ means that $v$ must be either a convex corner or a non-corner of $C$ as illustrated in Figs. 6(a) and (b). Label $L^{\prime}$ means that $v$ must be either a concave corner or a non-corner of $C$ as illustrated in Figs. 6(c) and (d). 


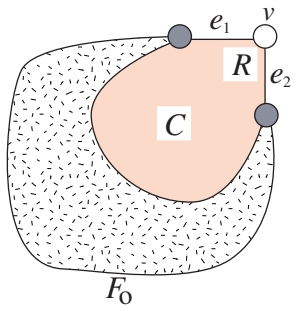

(a) A convex corner $v$ of $C$ and $F_{\mathrm{O}}$

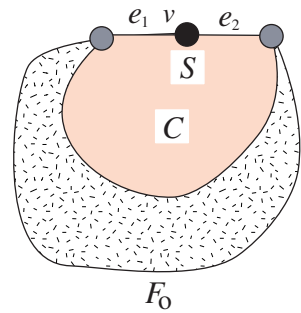

(b) A non-corner $v$ of $C$ and $F_{\mathrm{O}}$

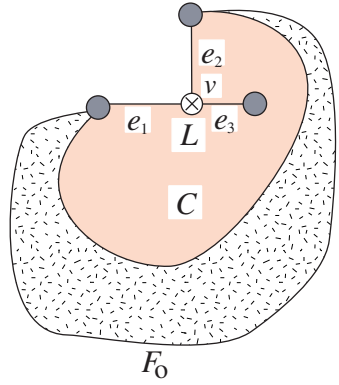

(c) A concave corner $v$ of $C$ and $F_{\mathrm{O}}$

Fig. 4. Illustration for Case 1.

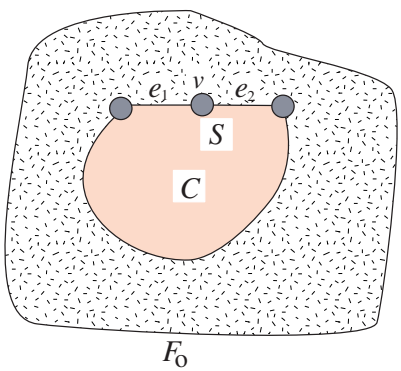

Fig. 5. Illustration for Case 2 .

Case 4: exactly one of $e_{1}$ and $e_{2}$ is an outer edge, and $d(v)=3$. (See Fig. 7.)

In this case, $v$ is an outer vertex, and the edge $e_{3}$ incident to $v$ other than $e_{1}$ and $e_{2}$ is an outer edge. By Condition (O-2), $v$ is either an $S$-vertex or an $L$-vertex of $F_{\mathrm{o}}$. Label $\lambda(C, v)$ is defined as follows:

$$
\lambda(C, v)=\left\{\begin{array}{l}
R \text { if } v \text { is an } S \text {-vertex of } F_{\mathrm{o}} \\
R^{\prime} \text { if } v \text { is an } L \text {-vertex of } F_{\mathrm{o}} .
\end{array}\right.
$$

Case 5: $d(v)=4$. (See Fig. 8.)

Let $e_{3}$ and $e_{4}$ be the edges incident to $v$ other than $e_{1}$ and $e_{2}$, then $\lambda(C, v)$ is defined as follows:

$$
\lambda(C, v)=\left\{\begin{array}{l}
R \text { if both } e_{3} \text { and } e_{4} \text { are outside } C \\
S \text { if exactly one of } e_{3} \text { and } e_{4} \text { is outside } C \\
L \text { if both } e_{3} \text { and } e_{4} \text { are inside } C
\end{array}\right.
$$

Clearly the following lemma holds.

Lemma 1. If $G$ has an extended rectangular drawing with designated corners, then the following (a)-(e) holds for any cycle $C$ of $G$ and any vertex $v$ of $C$ : 


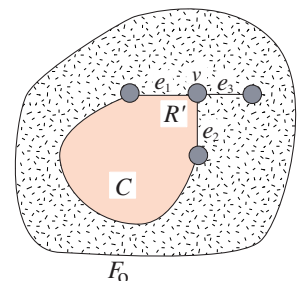

(a)A convex corner $v$ of $C$

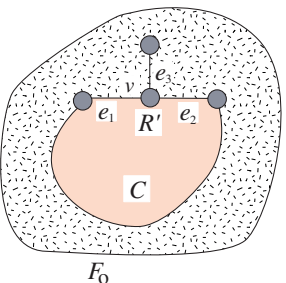

(b) A non-corner $v$ of $C$

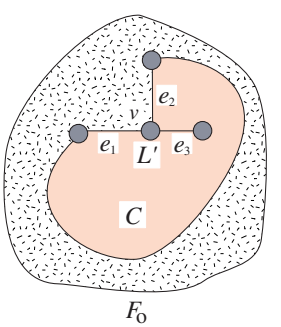

(c) A concave corner $v$ of $C$

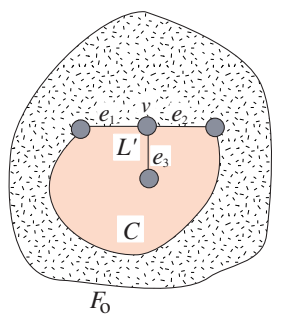

(d) A non-corner $v$ of $C$

Fig. 6. Illustration for Case 3.

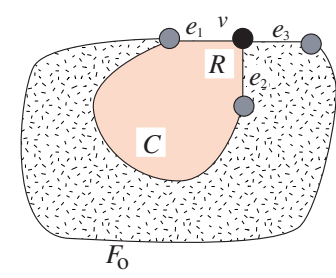

(a) A convex corner $v$ of $C$

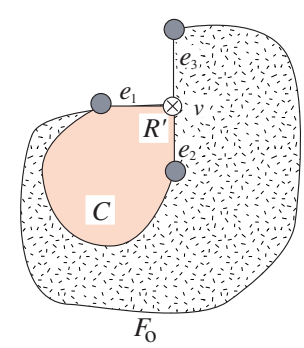

(b) A convex corner $v$ of $C$

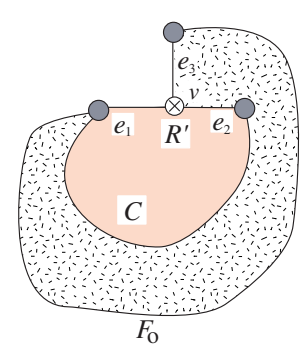

(c) A non-corner $v$ of $C$

Fig. 7. Illustration for Case 4.

(a) if $\lambda(C, v)=R$, then $v$ is a convex corner of $C$.

(b) if $\lambda(C, v)=S$, then $v$ is a non-corner of $C$.

(c) if $\lambda(C, v)=L$, then $v$ is a concave corner of $C$.

(d) if $\lambda(C, v)=R^{\prime}$, then $v$ is either a convex corner or a non-corner of $C$.

(e) if $\lambda(C, v)=L^{\prime}$, then $v$ is either a concave corner or a non-corner of $C$.

According to $\lambda(C, v)=R, S, L, R^{\prime}$ and $L^{\prime}$, we call $v$ an $R-, S-, L-, R^{\prime}$ - and $L^{\prime}$-vertex of cycle $C$, respectively. Let $n_{R}(C)$ be the number of $R$-vertices of cycle $C$. Define $n_{L}(C), n_{R^{\prime}}(C)$ and $n_{L^{\prime}}(C)$ similarly. One can easily know from Definition 1 that the following lemma hold.

Lemma 2. (a) $n_{R^{\prime}}\left(F_{\mathrm{o}}\right)=n_{L^{\prime}}\left(F_{\mathrm{o}}\right)=0$ for the outer face $F_{\mathrm{o}}$ of $G$.

(b) $n_{L}(F)=n_{L^{\prime}}(F)=0$ for any inner face $F$ of $G$.

(c) If $v$ is an inner vertex and $\lambda(C, v)=R$ or $L$ for a cycle $C$, then $d(v)=4$.

\section{$3 \quad$ Extended Rectangular Drawing}

In this section, we prove the following main theorem.

Theorem 1. A plane biconnected graph $G$ has an extended rectangular drawing with designated corners if and only if each cycle $C$ of $G$ satisfies

$$
n_{R}(C)-\left(n_{L}(C)+n_{L^{\prime}}(C)\right) \leq 4
$$




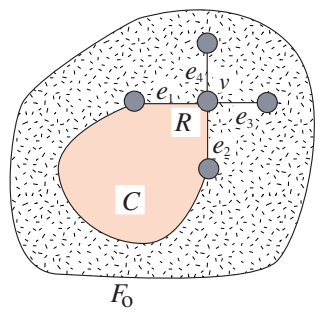

(a) A convex corner $v$ of $C$

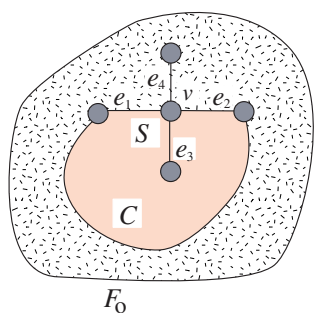

(b) A non-corner $v$ of $C$

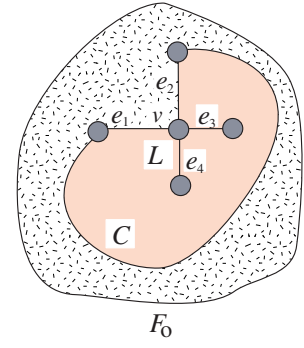

(c) A concave corner $v$ of $C$

Fig. 8. Illustration for Case 5.

and

$$
\left(n_{R}(C)+n_{R^{\prime}}(C)\right)-n_{L}(C) \geq 4 .
$$

One can derive Thomassen's condition as a corollary of Theorem 1. Although Corollary 1 below is slightly different from the presentation of Thomassen's condition [T84, Theorem 7.1], they are effectively equivalent with each other.

Corollary 1. Let $G$ be a plane biconnected graph of $\Delta \leq 3$. Assume that exactly four of the outer vertices of degree two are designated as $R$-vertices of $F_{\mathrm{o}}$, and all the other outer vertices are designated as $S$-vertices of $F_{\mathrm{o}}$, as illustrated in Fig. 1(a). Then $G$ has an extended rectangular drawing with designated corners, that is, a rectangular drawing if and only if each cycle $C$ of $G$ satisfies

$$
n_{R}(C)+n_{R^{\prime}}(C) \geq 4 .
$$

Proof. Omitted in this extended abstract due to the page limitation.

Before giving a proof of Theorem 1, we present some definitions and lemmas. A labeling $\psi_{C}$ of a cycle $C$ assigns a label $R, S$, or $L$ to each vertex $v$ of $C$. We say that $\psi_{C}$ is consistent with the labeling $\lambda$ of $G$ if

$$
\psi_{C}(v)=\left\{\begin{array}{lr}
R & \text { if } \lambda(C, v)=R ; \\
S & \text { if } \lambda(C, v)=S ; \\
L & \text { if } \lambda(C, v)=L ; \\
R \text { or } S \text { if } \lambda(C, v)=R^{\prime} \\
L \text { or } S \text { if } \lambda(C, v)=L^{\prime} .
\end{array}\right.
$$

We call a labeling of $C$ consistent with $\lambda$ simply a consistent labeling of $C$. We denote by $n_{R}\left(\psi_{C}\right)$ the number of vertices $v$ of $C$ such that $\psi_{C}(v)=R$, and denote by $n_{L}\left(\psi_{C}\right)$ the number of vertices $v$ of $C$ such that $\psi_{C}(v)=L$. If $\psi_{C}$ is a consistent labeling of $C$, then

$$
n_{R}(C) \leq n_{R}\left(\psi_{C}\right) \leq n_{R}(C)+n_{R^{\prime}}(C)
$$

and

$$
n_{L}(C) \leq n_{L}\left(\psi_{C}\right) \leq n_{L}(C)+n_{L^{\prime}}(C) .
$$

We then immediately have the following lemma. 
Lemma 3. If a plane graph $G$ has an extended rectangular drawing $D(G)$ with designated corners, then each cycle $C$ of $G$ has a consistent labeling $\psi_{C}$ such that

$$
n_{R}\left(\psi_{C}\right)-n_{L}\left(\psi_{C}\right)=4
$$

We are now ready to give a proof of the necessity of Theorem 1 , which is rather trivial.

Proof of the necessity of Theorem 1 Assume that $G$ has an extended rectangular drawing $D(G)$ with designated corners. By Lemma 3, each cycle $C$ of $G$ has a consistent labeling $\psi_{C}$ such that $n_{R}\left(\psi_{C}\right)-n_{L}\left(\psi_{C}\right)=4$. Since $\psi_{C}$ is a consistent labeling, by Eqs. (4) and (5) we have that $n_{R}(C) \leq n_{R}\left(\psi_{C}\right)$ and $n_{L}\left(\psi_{C}\right) \leq n_{L}(C)+n_{L^{\prime}}(C)$. We thus have

$$
n_{R}(C)-\left(n_{L}(C)+n_{L^{\prime}}(C)\right) \leq n_{R}\left(\psi_{C}\right)-n_{L}\left(\psi_{C}\right)=4,
$$

and hence $C$ satisfies Eq. (1). Similarly one can show that $C$ satisfies Eq. (2).

In the remainder of this section, we give a proof for the sufficiency of Theorem 1. The proof of the sufficiency is outlined as follows. First, we choose an appropriate inner face $F$ called a "corner face," as illustrated in Fig. 2(a). Next, we draw $F$ as a rectangle, and let $G^{*}=G-F$ be a graph obtained from $G$ by "trimming off" the face $F$ from $G$ as illustrated in Fig. 2(b). As shown in Lemma 5 later, every cycle of $G^{*}$ satisfies Eqs. (1) and (2). Repeating this operation, we can eventually obtain an extended rectangular drawing of $G$ with designated corners.

Before giving a proof of the sufficiency, we present some definitions and lemmas. Let $F$ be an inner face of $G$ containing an outer edge. Delete from $G$ all the outer edges on $F$, and then delete all resulting isolated vertices. The resulting graph is denoted by $G^{*}=G-F$.

We call an inner face $F$ of $G$ a corner face if $F$ satisfies the following four Conditions (a)-(d):

(a) at least one vertex of $F$ is designated as an $R$-vertex of $F_{\mathrm{o}}$. (Hence, the vertex is an outer vertex, and has degree two.)

(b) $2 \leq n_{R}(F) \leq 4$. (Hence, $F$ has two, three, or four $R$-vertices.)

(c) at least two of the $R$-vertices of $F$ are outer vertices. (Two of the $R$-vertices of $F$ are outer vertices in Fig. 9(a), three are outer vertices in Fig. 9(b), and four are outer vertices in Fig. 9(c).)

(d) $G^{*}=G-F$ is biconnected.

We have the following lemma.

Lemma 4. If each cycle $C$ of a plane biconnected graph $G$ satisfies Eqs. (1) and (2), then $G$ has a corner face.

Proof. Omitted in this extended abstract due to the page limitation. 


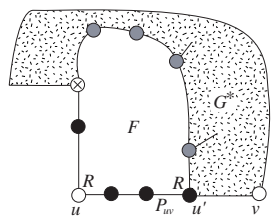

(a) Two outer $R$-vertices of $F$

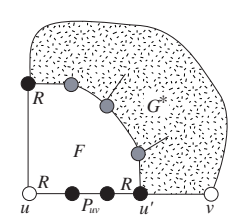

(b) Three outer $R$-vertices of $F$

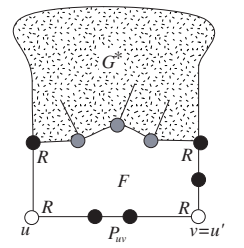

(c) Four outer $R$-vertices of $F$

Fig. 9. Illustration for a corner face $F$.

If $G$ has an extended rectangular drawing with designated corners, then by Lemma 3 each cycle $C$ has a consistent labeling $\psi_{C}$ such that $n_{R}\left(\psi_{C}\right)-n_{L}\left(\psi_{C}\right)=$ 4. We say that such a labeling $\psi_{C}$ is rectangular if $n_{R}\left(\psi_{C}\right)=4$ and $n_{L}\left(\psi_{C}\right)=0$. We then have the following lemma.

Lemma 5. If each cycle of $G$ satisfies Eqs. (1) and (2), then any inner face $F$ has a consistent rectangular labeling $\psi_{F}$.

Proof. Omitted in this extended abstract due to the page limitation.

Let $F$ be a corner face of $F$. Then, by Condition (d) of a corner face, $G^{*}=G-F$ is biconnected and hence the intersection of $G^{*}$ and $F$ is a single path $P^{*}$ of $G$, which is called the partitioning path of $F$. In Fig. 10, $P^{*}$ is drawn by thick lines. Clearly, the ends of $P^{*}$ are outer vertices and have degree three or four. All vertices of $P^{*}$ other than the ends are inner vertices of $G$. Since $F$ is an inner face of $G$, by Lemma $5 F$ has a consistent rectangular labeling $\psi_{F}$. We then decide the rough shape of the outer face $F_{\mathrm{o}}^{*}$ of $G^{*}$ according to $\psi_{F}$ : we designate each outer vertex $v$ of $G^{*}$ as a convex corner, a concave corner, or a non-corner, as follows. (See Fig. 10.)

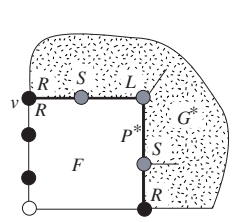

(a)

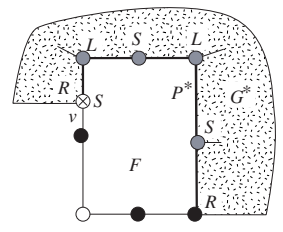

(b)

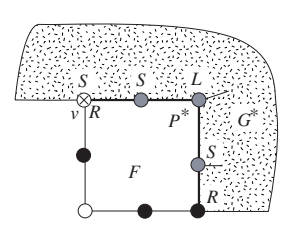

(c)

Fig. 10. Illustration for the designation of outer vertices of $G^{*}$.

[Definition 2] (Designation of outer vertices of $G^{*}$ )

Case 1: $v$ is not on $P^{*}$.

In this case, $v$ is an outer vertex of $G$. We designate $v$ as an $R-, S-$, and $L$-vertex of $F_{\mathrm{o}}^{*}$ if $v$ was designated as an $R$-, $S$ - and $L$-vertex of $F_{\mathrm{o}}$, respectively. Case 2: $v$ is a vertex of $P^{*}$ other than its ends. 
Since $\psi_{F}$ is rectangular, $\psi_{F}(v)$ is either $S$ or $R$. If $\psi_{F}(v)=S$ then we designate $v$ as an $S$-vertex of $F_{\mathrm{o}}^{*}$, while if $\psi_{F}(v)=R$ then we designate $v$ as an $L$-vertex of $F_{\mathrm{o}}^{*}$.

Case 3: $v$ is an end of $P^{*}$.

In this case, $v$ has degree three or four, and hence by Condition (O-2) $v$ is either an $S$-vertex of $F_{\mathrm{o}}$ or an $L$-vertex of $F_{\mathrm{o}}$.

Consider first the case where $v$ is an $S$-vertex of $F_{\mathrm{o}}$ as illustrated in Fig. 10(a). In this case, $v$ has degree three, and hence by Case 4 of Definition 1 , we have $\lambda(F, v)=R$. Since $\psi_{F}$ is consistent with $\lambda, \psi_{F}(v)=\lambda(F, v)=R$. Thus the vertex $v$ of polygon $D\left(F_{\mathrm{o}}^{*}\right)$ must have an inner angle $\pi-\pi / 2$, and hence we designate $v$ as an $R$-vertex of $F_{\mathrm{o}}^{*}$.

Consider next the case where $v$ is an $L$-vertex of $F_{\mathrm{o}}$ as illustrated in Figs. 10(b) and (c). Since $\psi_{F}$ is rectangular, either $\psi_{F}(v)=S$ or $\psi_{F}(v)=R$. If $\psi_{F}(v)=S$ as illustrated in Fig. 10(b), then we designate $v$ as an $R$-vertex of $F_{\mathrm{o}}^{*}$. If $\psi_{F}(v)=R$ as illustrated in Fig. 10(c), then we designate $v$ as an $S$-vertex of $F_{\mathrm{o}}^{*}$.

One can easily observe that the designation of the outer vertices of $G^{*}$ above satisfies Conditions (O-1)-(O-3). We have the following lemma.

Lemma 6. If every cycle of $G$ satisfies Eqs. (1) and (2), then any corner face $F$ of $G$ has a consistent rectangular labeling such that each cycle of $G^{*}=G-F$ satisfies Eqs. (1) and (2).

Proof. Omitted in this extended abstract due to the page limitation.

We are now ready to give a proof of the sufficiency of Theorem 1 .

Proof of the sufficiency of Theorem 1 We prove, by induction of the number $i$ of inner faces of $G$, the proposition that if every cycle of $G$ satisfies Eqs. (1) and (2) then $G$ has an extended rectangular drawing with designated corners.

If $i=1$, then $G=F_{\mathrm{o}}$ and by Lemma 2 and Eqs. (1) and (2), $n_{R}\left(F_{\mathrm{o}}\right)=4$ and $n_{L}\left(F_{\mathrm{o}}\right)=0$, and hence $G$ can be drawn as a rectangle having the four $R$ vertices of $F_{\mathrm{o}}$ as the convex corners. The drawing of $G$ is an extended rectangular drawing with designated corners. The proposition thus holds for $i=1$.

Assume inductively that $i \geq 2$ and the proposition holds for any plane graph of less than $i$ inner faces. We shall prove that the proposition holds for a plane graph $G$ of exactly $i$ inner faces. By Lemma $4 G$ has a corner face $F$, and by Lemma 6 6 has a consistent rectangular labeling $\psi_{F}$ for which each cycle of $G^{*}=G-F$ satisfies Eqs. (1) and (2). Since $\psi_{F}$ is rectangular, $F$ can be drawn as a rectangle $D(F)$ having the four $R$-vertices of $\psi_{F}$ as convex corners. Since the number of inner faces of $G^{*}$ is equal to $i-1$, by the inductive assumption $G^{*}$ has an extended rectangular drawing $D\left(G^{*}\right)$ with designated corners. The designation of outer vertices of $G^{*}$ in Definition 2 implies that one can combine the drawings $D(F)$ and $D\left(G^{*}\right)$ to an extended rectangular drawing of $G$ with designated corners. 


\section{Conclusion}

In this paper, we gave in Theorem 1 a necessary and sufficient condition for a plane graph to have an extended rectangular drawing with designated corners. It immediately yields a necessary and sufficient condition for a plane graph of $\Delta \leq 4$ to have an ordinary rectangular drawing. Thomassen's condition for a plane graph of $\Delta \leq 3$ to have a rectangular drawing can be easily derived from our condition as in Corollary 1. It is remaining to obtain an efficient algorithm to find an extended rectangular drawing with designated corners.

\section{References}

BETT99. G. Di Battista, P. Eades, R. Tamassia and I. G. Tollis, Graph Drawing, Prentice Hall, NJ (1999).

BS88. J. Bhasker and S. Sahni, A linear algorithm to find a rectangular dual of a planar triangulated graph, Algorithmica, 3, pp.247-278, 1988.

GT97. A. Garg and R. Tamassia, A new minimum cost flow algorithm with applications to graph drawing, Proc. of Graph Drawing'96, Lect. Notes in Computer Science, 1190, pp. 201-206, 1997.

H93. X. He, On finding the rectangular duals of planar triangulated graphs, SIAM J. Comput., 22, 6, pp. 1218-1226, 1993.

H99. X. He, On floor-plan of plane graphs, SIAM J. Comput., 28, 6, pp. 21502167, 1999.

KH97. G. Kant and X. He, Regular edge-labeling of 4-connected plane graphs and its applications in graph drawing problems, Theoret. Comput. Sci., 172, pp. 175-193, 1997.

KK84. K. Kozminski and E. Kinnen, An algorithm for finding a rectangular dual of a planar graph for use in area planning for VLSI integrated circuits, Proc. of 21st DAC, Albuquerque, pp. 655-656, 1984.

L90. T. Lengauer, Combinatorial Algorithms for Integrated Circuit Layout, Wiley, Chichester, 1990.

RNN02. M. S. Rahman, S. Nakano and T. Nishizeki, Rectangular drawings of plane graphs without designated corners, Computational Geometry, 21, pp. 121$138,2002$.

RNN98. M. S. Rahman, S. Nakano and T. Nishizeki, Rectangular grid drawings of plane graphs, Comp. Geom. Theo. Appl., 10(3), pp. 203-220, 1998.

T84. C. Thomassen, Plane representations of graphs, J. A. Bondy, U. S. R. Murty (Eds.), Progress in Graph Theory, Academic Press Canada, Don Mills, Ontario, Canada, pp. 43-69, 1984. 